\title{
Diacronie
}

Studi di Storia Contemporanea

$N^{\circ} 7,3 \mid 2011$

«Spagna Anno Zero»: la guerra come soluzione

\section{Solidarité Internationale Antifasciste, ou l'humanitaire au service des idées anarchistes}

\section{Valentin Cionini}

\section{(2) OpenEdition}

Journals

Édition électronique

URL : http://journals.openedition.org/diacronie/3311

DOI : 10.4000/diacronie.3311

ISSN : 2038-0925

Éditeur

Association culturelle Diacronie

Référence électronique

Valentin Cionini, «Solidarité Internationale Antifasciste, ou l'humanitaire au service des idées anarchistes », Diacronie [En ligne], N 7, 3 | 2011, document 13, mis en ligne le 29 juillet 2011, consulté le 19 avril 2019. URL : http://journals.openedition.org/diacronie/3311 ; DOI : 10.4000/diacronie.3311 


\title{
Diacronie
}

N. 7 | 7|2011 Spagna Anno Zero: la guerra come soluzione

\section{3/}

\section{Solidarité Internationale Antifasciste, ou l'humanitaire au service des idées anarchistes}

\author{
Valentin CIONINI*
}

En temps de guerre, le soutien aux populations civiles victimes du conflit ou aux combattants blessés, a longtemps été le fait de congrégations (Pères Blancs, Ordre de Saint Jean de Malte) répondant à des motivations religieuses, selon le principe de la charité chrétienne. La Croix-Rouge, organisation se voulant neutre, apporte aussi un soutien de type "humanitaire" aux victimes des conflits. La montée de la question sociale bouleverse peu à peu ce modèle caritatif et religieux et la guerre civile espagnole est une étape décisive dans l'évolution de cette aide. C'est en effet durant ce conflit que l'humanitaire prends un tournant politique et que le caritatif laisse sa place à un soutien idéologique. L'action déployée par le Secours Rouge International (SRI), créé par l'Internationale Communiste (IC) en 1923, en faveur des républicains espagnols, est sûrement l'acte humanitaire le plus connu du conflit, mais il ne doit pas occulter la solidarité en faveur des anarchistes espagnols qui a été bien réelle. La Confederación General del Trabajo (CNT) et la Federación Anarquista Ibérica (FAI) ont bénéficié durant tout le conflit d'une aide au départ dispersée, qui prend la forme courant 1937 d'une organisation de solidarité internationale structurée, Solidaridad Internacional Antifascista (SIA). Conçue dès le départ comme une organisation humanitaire ayant pour but de soutenir le mouvement antiautoritaire espagnol, SIA est surtout pendant tout le conflit un moyen de diffusion des idées anarchistes. 


\section{La naissance de Solidaridad Internacional Antifascista et son développement}

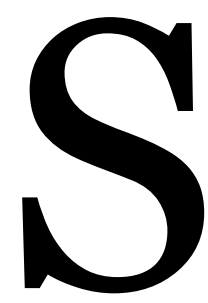

olidarité Internationale Antifasciste (SIA) naît en Espagne en juin 1937. La CNT, qui est à l'origine avec les autres organisations libertaires espagnoles (FAI, Federación Ibérica de Juventudes Libertarias) de cette organisation, l'a conçue comme une réponse anarchiste à la mainmise des communistes et de leurs organisations (SRI notamment) sur la vie publique et politique lors de la guerre d'Espagne. De par ses objectifs "humanitaires", mais aussi politiques, la SIA est une organisation complexe. La création de la SIA répond à l'inquiétude de la CNT devant le développement des organisations communistes en Espagne et les tensions qui en découlent (les journées de mai 1937 à Barcelone en sont l'exemple le plus frappant ${ }^{1}$ ). Autre élément décisif à l'origine de la création de la SIA : la faiblesse du mouvement libertaire hors d'Espagne et donc le manque d'appuis internationaux pour la CNT, que cela soit sur un plan politique, idéologique ou humanitaire.

La décision officielle de créer une organisation de solidarité internationale est prise par le plénum national de la CNT à Valence le 15 avril 1937². C'est Pedro Herrera, alors secrétaire du comité péninsulaire de la FAI, qui en fait mention pour la première fois :

Dans le rapport approuvé avec de légères variations, qui a été fait à la suite du second point de l'ordre du jour du plénum cité et dans son paragraphe c) du troisième point, il est dit: «Se consacrer avec ardeur à connaître en détail le développement économique du SRI, en prenant en compte qu'il existe chez nous la conviction fondée, que les recettes qu'ils mènent à bien, servent exclusivement pour leurs funestes propagandes, toute intention de solidarité étant absente de leur esprit. [...] En examinant cet aspect du problème, le moyen d'affaiblir cette organisation qui sert à alimenter économiquement le Parti Communiste, est la constitution immédiate d'un organe d'aide ou de solidarité au sein de notre organisation. » En plus d'être accepté, ce rapport se complète en suggérant son

1 LORENZO, César Marcel, op. cit, pp. 343-344; MORROW, Felix, Révolution et contre révolution en Espagne (1936-1938), Paris, La brèche, 1978, pp. 101-129.

2 International Institute of Social History, Amsterdam, microfilm CNT n ${ }^{\circ} 64 \mathrm{C}$, abrégé dans la suite de l'article en IISG, CNT 64C et suivant. 
nom à l'organisation de solidarité à créer: Solidarité pour les Victimes du Fascisme 3

La SIA ne répond ainsi pas qu'à un objectif humanitaire, mais s'inscrit dans un champ politique plus global. La CNT met sur pied une véritable stratégie politique. Dès le départ, cette organisation est impulsée par le Mouvement Libertaire Espagnol (Movimiento Libertario Español ; MLE) dans le but de contrer la montée en puissance du Parti Communiste espagnol (PCE) et de l'organisme de solidarité de l'IC, le SRI. Alors que le PCE n'est qu'un petit parti au début de la guerre, l'aide de l'URSS et de l'IC va le transformer en un acteur fondamental de la vie politique espagnole.

La faible influence du mouvement libertaire hors des frontières espagnoles et le besoin vital qu'a la CNT de trouver des soutiens à l'étranger pour contrer politiquement l'IC et améliorer son approvisionnement en armes, matériel médical, vivres, sont aussi des facteurs prépondérants dans la création de la SIA. Même si la CNT fait partie d'une organisation internationale, l'AIT, cette dernière n'a que très peu d'influence et de marge de manœuvre pour l'aider. Les querelles doctrinaires internes au mouvement libertaire inquiètent et agacent la CNT dès octobre 1936. Cette exaspération culmine lors du congrès de l'AIT en décembre 1937 à Paris. Les Espagnols mettent donc en avant la constitution de la SIA comme moyen d'augmenter l'audience du mouvement libertaire dans le monde. Les déclarations de la CNT lors de la conférence internationale de l'AIT de décembre 1937 sont sans équivoque sur le besoin politique qu'a la CNT de sortir de son isolement international:

Nous avons constitué la Solidarité Internationale Antifasciste (SIA), organisme qui peut très bien être le véhicule qui facilite un futur agrandissement de notre centrale anarcho-syndicaliste, de la même forme que le Secours Rouge International l'a fait pour l'Internationale Communiste 4 .

3 IISG, CNT 64C, « En el dictamen aprovado con ligeras variaciones, que se elaboró a consecuencia del segundo punto del orden del día del citado pleno y en su apartado c) del punto tercero, dice “ Dedicarse con afán a conocer en detalle el desenvolvimiento económico del SRI, teniendo en cuenta que en nosotros existe el convencimiento fundado de que las recaudaciones que llevan a cabo, sirven exclusivamente para sus propagandas funestas, estando ausente de su ánimo toda intencion solidaria [...] Al examinar este aspecto del problema, como medio de debilitar este organismo que sirve para alimentar economicamente al partido communista, la constitución inmediata de un órgano de ayuda o solidaridad en seno de nuestra organización." Además de aceptado esta dictamen, se completa sugiriendo se llama, el organismo de solidaridad a crear : Solidaridad pro Victimas del Fascismo.».

4 IISG, CNT 36B, « Hemos constitudio la Solidaridad Internacional Antifascista, (SIA) organismo que muy bien puede ser el vehículo que facilite un futuro engrandecimiento de nuestra central anarcosindicalista, de igual forma que el Socorro Rojo Internacional lo ha facilitado a la Internacional Communista ». 
Lors de ce congrès, la position de la CNT est délicate vis-à-vis de ses organisations sœurs. En effet depuis le début de la guerre civile, certaines sections, notamment la Confédération Générale du Travail Syndicaliste Révolutionnaire (CGTSR), organisation française, critiquent fortement l'action de la CNT dans la guerre et ses participations aux gouvernements catalan et républicain 5 . Cette situation est inacceptable pour la CNT, qui se voit obligée de justifier ses actions devant l'AIT. La SIA est un moyen de montrer qu'elle œuvre à la diffusion nationale et internationale des idées libertaires, tout en mettant la pression sur les sections de l'AIT qui critiquent son action. Au sein de l'internationale anarcho-syndicaliste, la seule autre organisation capable d'égaler la CNT (du moins en activité rapportée à la population) est la Sveriges Arbetares Centralorganisation (SAC) suédoise, qui va grandement favoriser la création internationale de la SIA et apporter son soutien à l'action de la CNT en Espagne en demandant à ce que les organisations libertaires, même si elles ont des griefs à formuler contre la CNT ou la FAI, se gardent de toutes critiques publiques à l'égard de ces deux structures ${ }^{6}$. Les critiques au sein de l'AIT envers son membre le plus important, qui plus est dans une période révolutionnaire et de guerre, prouvent la faiblesse internationale du mouvement libertaire, qui n'en finit pas de se déchirer sur le plan doctrinaire, entre "puristes" et "réformistes" supposés. C'est pourquoi la CNT décide de se rapprocher de groupes et d'organisations prolétariennes non spécifiquement anarchistes qui ne «veulent rien savoir de l'AIT »7.Cette volonté d'élargissement de l'audience internationale de la CNT est marquée lors du même congrès de l'AIT par un point de l'ordre du jour sollicité par la CNT : la nécessité de former un front antifasciste prolétarien pour vaincre le fascisme mondial ${ }^{8}$. L'idée d'un front unique prolétarien destiné à écraser le fascisme mondial n'est pas sans rappeler la stratégie de l'Union Anarchiste (UA) en France, qui prône l'idée d'un front révolutionnaire, opposé au Front Populaire9. A côté de cette orientation stratégique censée développer le mouvement libertaire au niveau international et qui ne concerne que la France et dans une moindre mesure l'Espagne, l'AIT reste sur des positions

5 Voir MAITRON, Jean, Le mouvement anarchiste en France, t. II: de 1914 à nos jours, Paris, François Maspero, 1975, pp. 33-35.

6 IISG, CNT 36B.

7 Ibidem.

8 Ibidem.

9 David Berry estime que l'attitude de Front Révolutionnaire a été très profitable au mouvement libertaire français et notamment à l'UA, dont le nombre de membres passe à presque 4500 en 1937 contre moins de 2.000 en 1936 et dont le journal, « Le Libertaire », passe d'un tirage hebdomadaire de 5.000 exemplaires à plus de 25.000 au printemps 1937. Voir BERRY, David, L'anarchisme français et la révolution espagnole 1936-1939, Paris, Alternative Libertaire, 2001, p. 7. 
stratégiques qui ne concernent que le mouvement anarcho-syndicaliste et que la CNT désapprouve $^{10}$. Lors de la préparation à ce congrès de l'AIT, qui se tient à Paris du 6 au 12 décembre 1937 à la Mutualité, on assiste à des échanges de points de vue assez violents entre la CNT et la CGTSR qui voit d'un mauvais œil la participation gouvernementale de la CNT, ainsi que sa volonté d'élargir le cercle des soutiens à des organisations non libertaires. Cette passe d'armes culmine avec des accusations de "déviationnisme" envers la CNT portées par la CGTSR" ${ }^{11}$ La présentation dans le même congrès par la CNT à la fois de la SIA et de son idée de front antifasciste prolétarien, n'est pas un hasard. Les seules forces libertaires ne suffisent pas à assurer à la CNT un soutien suffisamment important. Les oppositions internes au mouvement libertaire affaiblissant la position de la CNT en Espagne, il faut donc aller chercher de l'aide du côté des révolutionnaires anti-autoritaires. La CNT cherche à sortir de cette situation en demandant aux organisations avec lesquelles elle entretient des liens privilégiés d'élargir la base du soutien à l'Espagne antifasciste. En France, Le Libertaire du 16 octobre reproduit une lettre d'Horacio Prieto ${ }^{12}$ du 3 octobre, qui demande aux camarades « $[\ldots]$ dans le but de nous soutenir, d'en appeler à quiconque ${ }^{13}$. Toujours en France, Alfonso de Miguel, membre dirigeant de la CNT, fait publier en Une du Libertaire du 2 octobre 1936 un appel qui montre l'agacement de la CNT face aux querelles doctrinales qui isolent les différentes composantes du mouvement libertaire et empêchent une aide efficace :

\begin{abstract}
A l'heure actuelle - octobre 1936 - celui qui en Espagne, en France ou dans n'importe quel pays du monde ne sait pas oublier les petites différences doctrinales ou les querelles purement personnelles pour forger l'unité révolutionnaire de toutes les forces ouvrières et socialement démocratiques n'aura plus désormais le droit de s'appeler révolutionnaire ${ }^{14}$.
\end{abstract}

Pedro Herrera ${ }^{15}$ qui quitte ses fonctions au sein de la FAI pour prendre en charge le secrétariat de la SIA rappelle à de nombreuses reprises dans ses rapports ou ses

10 Voir IISG, CNT 36B.

11 Ibidem.

12 Horacio Martinez Prieto (1902-1985), secrétaire confédéral de la CNT à partir de 1936.

13 Le Libertaire, 16 octobre 1936.

14 Alfonso de Miguel, Le Libertaire, 2 octobre 1936.

15 Pedro Herrera Camarero (1909-1969), cheminot, responsable du commerce au conseil d'économie de la Généralité de Catalogne en octobre 1936, il devient conseiller à la santé publique du gouvernement de la Généralité de Catalogne de décembre 1936 à avril 1937. Il est l'un des signataires le 25 décembre 1936, du décret autorisant l'avortement. Secrétaire du comité Péninsulaire de la FAI, il est aussi nommé secrétaire du Comité national de la SIA. 
courriers que c'est le mouvement libertaire qui a permis la création de la nouvelle organisation de solidarité, mais que celle-ci doit être ouverte à tous les véritables militants révolutionnaires, sans esprit de parti ${ }^{16}$.

La création de la SIA, une fois votée et décidée par la CNT doit commencer par la mise en place des sections locales, tout d'abord en Espagne. Dès le 5 juin 1937, Pedro Herrera demande aux organisations du mouvement libertaire qu'elles donnent des consignes à leurs membres pour aider à la constitution de la SIA et pour qu'ils y adhèrent en masse ${ }^{17}$. La constitution des groupes locaux de la SIA se fait presque systématiquement sur le même modèle. Après que la CNT et le MLE se soient saisis de la question de la formation de groupes de la SIA et qu'ils aient répercuté leurs consignes d'adhésion à leurs militants, ces derniers se mettent peu à peu en contact, le plus souvent regroupés au sein d'un comité de solidarité préexistant, avec le comité régional, ou national s'il n'existe pas, de la SIA, et demandent lors d'une assemblée générale, l'adhésion de leur groupe à la nouvelle structure de solidarité. Une fois le groupe local légalement constitué, il s'efforce d'intégrer en son sein les autres groupes de solidarité, ou leurs militants, qui peuvent exister dans leur localité, de manière à ce qu'il ne reste plus sur la ville que deux organisations de solidarité et d'entraide : le SRI et la SIA.

Les responsables de la SIA savent ce qu'ils veulent et ont déjà un plan préétabli sur le modèle de développement de l'organisation. Pourtant, sans l'appui du mouvement libertaire, notamment de la CNT, la SIA n'aurait jamais pu prendre son essor et serait restée une petite organisation oppositionnelle au SRI. Créée par la CNT, elle n'existe véritablement que par la volonté du MLE. On retrouve cette tendance en France.

\section{SIA, une organisation humanitaire}

La SIA a été créée comme moyen politique au service du MLE. Pourtant cela ne doit pas occulter la dimension indéniablement "humanitaire" que revêt son action durant la guerre d'Espagne. Cette appellation d' "humanitaire" est anachronique, appliquée à cette période historique, mais correspond pourtant bien à l'activité de solidarité que la SIA mène entre juin 1937 et janvier 1939. La dimension humanitaire de l'action de la SIA évolue entre sa création et la fin de son activité. La nécessité d'une organisation de solidarité, entrevue sous un angle politique par la CNT, devient, au fil du temps et des différentes étapes du conflit, une nécessité pour la population civile, mais aussi pour les

16 Voir IISG, CNT 87C, CNT 50B, CNT 100F.

17 Voir IISG, CNT 87C. 
combattants du Front. Cela emmène la SIA à effectuer ce que nous appellerons son "tournant humanitaire". Une fois entrée dans cette "logique humanitaire", l'organisation doit justifier de son rôle et requalifier ses fondements idéologiques, pour effectuer un véritable travail d'aide et de secours.

Si l'on peut parler d'une véritable "entrée" de la SIA sur le plan de l'aide "humanitaire" dans le camp républicain, c'est parce qu'elle développe très rapidement après sa création en juin 1937, un effort sur le terrain afin de pallier le manque d'efficacité des organismes déjà en place, que cela soit pour des raisons politiques ou autres. La SIA est donc obligée par les circonstances de jouer pleinement son rôle d'organisation "proto-humanitaire". Elle est confrontée de ce fait à une réalité du terrain qui est tout autre que celle qu'ont pu envisager la CNT et le MLE au moment de sa création.

Les sections hôpital et sanitaire de la SIA sont les premières à entrer en lice, tant au Front qu'à l'arrière. Sur le front, la prise en compte des besoins sanitaires est primordiale pour que le travail effectué soit efficace. La première action de la SIA consiste donc à être reconnue par l'Etat-major républicain dont le soutien est indispensable à son intervention. Pour ce faire, elle écrit au gouvernement central, qui réside alors à Valence, et aux autorités régionales catalanes représentées par la Généralité de Catalogne, afin de les informer de l'initiative qu'elle a prise de mettre en place des brigades de sauvetage au Front ${ }^{18}$. Ces brigades doivent assurer le transport des blessés des zones de combat à l'arrière, et apporter les premiers secours aux combattants. Elles sont composées de volontaires de la SIA, médecins, infirmières, brancardiers, qui sont épaulés par une ambulance par brigade ${ }^{19}$. Le nombre exact de ces brigades ne nous est pas connu, mais il doit être suffisamment important et leur action efficace, puisque le gouvernement de Valence se félicite de leurs interventions à plusieurs reprises entre juillet 1937 et novembre $1938^{20}$. Cette action sur le Front, se double d'une action à l'arrière en faveur des combattants. Ainsi une polyclinique spécialisée dans la rééducation des invalides de guerre est-elle mise en place à Jaén durant l'été $1937^{21}$. Cet établissement a pour but d'aider les combattants blessés, toutes tendances politiques confondues, et qui ne peuvent repartir au Front, à trouver un travail à la sortie de leur convalescence, par le biais du réseau de solidarité de la SIA. Un hospice pour les blessés de guerre est également créé à Gandía dans la région de Valence. Il accueille environ 80 anciens combattants. Enfin, la SIA gère en commun

8 Voir courrier du 30 juin 1937, IISG, CNT 64C.

Voir IISG, CNT 64C.

Voir IISG, CNT 64C et IISG, CNT 87C.

1 Voir IISG, CNT 64C. 
avec la CNT quatre hôpitaux militaires (appelés hospitales de sangre en espagnol) en Catalogne. Ces hôpitaux accueillent les combattants blessés au Front et évacués par les brigades de secours de la SIA. Leur approvisionnement est assuré par l'organisation, qui prend en charge aussi le transport des vivres et matériel qui y sont acheminés. De cette manière, la SIA peut garantir que les blessés ont une alimentation adaptée à leur état de santé22.

En ce qui concerne l'aide des sections sanitaires et hospitalières aux civils, elle est surtout marquée par la constitution d'une brigade de volontaires équipés de matériel médical et de recherche pour porter secours aux civils victimes de bombardements ${ }^{23}$. La création de cette unité témoigne de l'ampleur du traumatisme résultant des bombardements de Durango et Guernica et répond à l'éventualité d'avoir encore à faire ce type d'intervention d'urgence.

La SIA met également en place dans les villes, essentiellement de Catalogne, où elle est présente ${ }^{24}$, des services de médecine en direction des civils, avant tout tournés vers l'action sociale. Ainsi à Vich par exemple, c'est une quinzaine d'équipes, pour une population qui avoisine les 16 ooo habitants, qui effectuent des tournées médicales dans les quartiers de la ville afin de s'enquérir des besoins des malades, blessés, ou personnes âgées. Ces équipes sont secondées par un dispensaire médicopharmaceutique, qui délivre gratuitement des médicaments ${ }^{25}$.

L'action menée par les services hospitaliers et sanitaires prend indéniablement en compte les besoins qui ont été constatés durant la première année de guerre, mais aussi les progrès qui sont inhérents à toute guerre. Les unités médicales mobiles créées sur le front participent de ces avancées. Pourtant, ce n'est pas par l'action médicale que va se distinguer la SIA, mais bien plus par l'action sociale.

C'est le service d'action sociale de la SIA qui effectue la plus grosse partie du travail de solidarité de l'organisation. Son action est multiple: envoi de colis pour les combattants et les civils, ouverture et gestion de lieux publics (casa de dormir, qui correspondent à des foyers d'hébergement, restaurant du milicien, restaurants populaires, etc.), mais aussi distribution de nourriture, de vêtements, de repas. Cette action sociale est large et dépend des sections locales qui l'organisent selon les besoins et les forces disponibles.

22 Ibidem.

23 Ibidem.

24 Des exemples sont donnés pour Vich, Manresa, Barcelone, Gérone, Figueres, Tarragone et Puigcerdá. Voir IISG, CNT 100B.

25 La SIA précise bien, d'ailleurs, que l'ensemble de ses prestations médicales sont gratuites et qu'elles sont le fait de bénévoles et financées par l'aide internationale. Voir IISG, CNT 100B. 
Dès le début, l'une des premières activités est l'envoi de colis au Front et à l'arrière. Cette action correspond à la nécessité de distinguer les besoins des combattants et des civils et de répondre à ceux nés de la guerre. Pour essayer de canaliser les dons venant de l'étranger et éviter l'envoi de colis inutiles ${ }^{26}$, la SIA met en place des paquets standards de nourriture ou de vêtements avec des contenus adaptés aux besoins des combattants, des civils ou des enfants. Ce sont les donateurs qui choisissent eux-mêmes la nature du colis qu'ils vont constituer et donc la catégorie de bénéficiaires qui seront concernés. La constitution de ces colis est l'un des volets par lesquels la SIA a fait la preuve de son efficacité. Faciles à transporter depuis l'étranger, ils transitent également aisément en Espagne, depuis les centres de stockage vers les destinataires. L'un des autres avantages que comporte ce type d'action met en jeu la question de la conservation. En effet, les colis ne contiennent que des aliments de première nécessité, hautement nutritifs et qui ont une longue durée de conservation ${ }^{27}$. Ce système de colis standard rationalise donc l'aide alimentaire et lui donne une plus grande efficacité. De la même façon, des envois standards de vêtements sont mis en place, le contenu changeant selon les saisons, le sexe, l'âge et la distinction combattant/civil ${ }^{28}$. L'organisation de ce service des "paquets" s'organise autour de trois principes: recevoir, transporter, distribuer. Pour recevoir, la SIA met en place quinze centres de collecte, qui réceptionnent autant les dons locaux qu'internationaux ${ }^{29}$ et qui les répartissent ensuite en fonction des besoins.

Outre la gestion de ce service des paquets, les sections locales développent un grand nombre d'activités sur place. Ainsi, à Vich et Ausona, à l'automne 1937, ce sont des déjeuners pour les enfants qui sont servis tous les jours entre 8 et 9 heures. Devant le succès de cette initiative, le conseil national de la SIA demande à l'ensemble de ses sections de mettre en place de telles distributions sur l'ensemble du territoire républicain ${ }^{30}$. En parallèle, des casas de dormir sont créées un peu partout, à Valence,

26 Un sympathisant anglais envoie ainsi deux caisses de champagne et de caviar pour que les combattants fêtent noël 1937, alors que beaucoup manquent de vêtement ou de pain. Voir IISG, CNT 101D.

27 Pour exemple, l'un des colis standard destiné au Front contient 1 kilo de riz, 1 kilo de sucre, 1 kilo d'haricots, 1 kilo de pâtes et un colis destiné à un enfant contient 6 boites de lait concentré en plus. Voir IISG, CNT 101D.

28 Un colis pour un combattant durant l'hiver, se compose d'un manteau, d'une écharpe et d'un pull. Celui qui est destiné à un enfant l'été, contient deux chemises, des sandales et un short. Voir IISG, CNT 101D.

29 Ces centres sont situés à Port-Bou, Gérone, Barcelone, Tarragone, Lérida, Castellon, Valence, Alicante, Carthagène, Almeria, Cabeza del Buey, Jaén, Albacete et Madrid. Il est intéressant de remarquer que certains centres (ceux d'Albacete, Cabeza del Buey, Madrid), sont situés très proche de la ligne de Front. Voir IISG, CNT 64C.

30 Voir IISG, CNT 64C. 
Barcelone, Jaén, Tarragone, pour permettre aux combattants en permission dans ces villes, ou aux réfugiés d'autres régions d'Espagne qui fuient les combats, de s'abriter. Rien qu'à Valence il existe neuf établissements de ce type, qui peuvent accueillir près de 500 personnes ${ }^{31}$. La création de ces établissements correspond à un besoin. Face aux déplacements de populations qui fuient les combats et l'avancée des troupes nationalistes, les grandes villes de la zone républicaine voient un nombre croissant de réfugiés arriver. Lorsque les structures en place et la solidarité locale n'arrivent plus à absorber ce flux, la création de nouveaux centres est indispensable, d'où l'institution de ces casas de dormir. Assez similaires, les restaurants populaires se chargent de préparer et distribuer des repas. Situés dans des localités où l'influence de la SIA est forte, ils permettent aux réfugiés et aux combattants en permission de manger et d'avoir un lieu d'accueil de jour, les casas de dormir ne fonctionnant que la nuit. Celui de Valence sert environ 600 repas par jour, celui de Murcie 400, ceux de Puigcerdá, la Seu d'Urgell et de Prat de Llobregat 200.

Début 1938, la SIA gère dans toute la zone républicaine, sept restaurants populaires (Valence, Murcie, une ville indéterminée, Prat del Llobregat, Puigcerdá, la Seu d’Urgell, Andujar), trois auberges (Onteniente, Godella et une ville indéterminée), trois restaurants du milicien (un à Valence et deux à Barcelone), deux ateliers de confection (Manzanares et une ville indéterminée), quatre refuges pour sans abris (tous à Valence), un hospice pour blessés de guerre (Gandía), une vingtaine de casas de dormir (neuf à Valence, huit à Barcelone, une à Jaén, deux à Tarragone)32. Ces différentes actions, permettent à la SIA d'être considérée comme une organisation de solidarité qui compte en Espagne. L'entrée dans le champ de l'humanitaire achève de transformer la SIA en véritable organisme d'aide et de secours aux victimes de la guerre dès l'automne 1937. L'organisation en partenariat avec le SRI de deux campagnes - celle de l'hiver 1937-1938 et celle qui est intitulée campagne pour les enfants, toujours lors du deuxième hiver du conflit - permet de mesurer combien la SIA est désormais détachée de la logique idéologique qui a entraîné sa création.

\section{SIA, une organisation internationale}

Organisation politico-humanitaire, ayant une action non-négligeable en Espagne, la SIA devient-elle pour autant une organisation internationale, répondant aux attentes

\footnotetext{
31 Ibidem.

32 Pour les listings des établissements de la SIA, excepté les colonies d'enfants, voir IISG, CNT 64C, $100 \mathrm{~B}$ et $100 \mathrm{~F}$.
} 
du MLE? La fondation de la SIA répond, on l'a vu, à des objectifs politiques et humanitaires, qui évoluent dans le temps, en fonction des besoins et de la situation. Les objectifs du MLE en ce qui concerne la question internationale sont complexes et la situation politique ne joue pas en leur faveur : guerre de tendances entre anarchosyndicalistes, individualistes, communistes-libertaires, faible implantation internationale, faible poids politique. Sans vouloir être une internationale anarchiste, la SIA doit rassembler les libertaires et d'autres tendances révolutionnaires pour résoudre une partie de ces problèmes et aider les anti-autoritaires engagés dans la guerre civile.

Décréter la création d'une organisation de solidarité internationale est une chose, l'implanter réellement dans des pays où le mouvement libertaire est faible, voir inexistant, en est une autre. De la création de la SIA, en juin 1937, aux premiers mois de 1939, un certain nombre de sections étrangères voient le jour. Si le cas de la France, où la CNT demande aux militants de l'UA de transformer le Comité pour l'Espagne Libre (CEL) en section française, est un peu particulier, les autres sections ne sont pas mises en place de cette manière, qui repose sur la préexistence d'une organisation de solidarité d'obédience libertaire déjà structurée. La création de ces nouvelles sections s'étale dans le temps, et n'est pas régulière. Les premières sections à être créées, avant la fin 1937, sont les sections française, suédoise, britannique, portugaise et nordafricaine. La section nord-africaine ne concerne pas l'Algérie française, le Maroc ou la Tunisie, mais la partie espagnole du Maroc, d'où une partie de la rébellion nationaliste est partie. Elle est créée par des militants de la CNT entrés en clandestinité et qui se réfugient dans les territoires français pour échapper aux troupes nationalistes ${ }^{33}$. C'est la même chose pour la section portugaise, créée par des militants de la FAI34 (la FAI réunit des militants anarchistes espagnols et portugais au sein d'une fédération ibérique) qui sont en Espagne, elle ne peut avoir qu'une activité clandestine dans le Portugal de Salazar et ses membres n'ont pas de véritable activité en faveur de la SIA dans leur pays d'origine. Même si elles existent sur le papier, la création de ces sections est donc plus un effet d'annonce de la part de la SIA qu'une existence et une implantation réelle.

Mis à part les cas français, portugais et nord-africain, les premières sections créées ont un mode de construction assez similaire : un militant anarchiste (ou une militante) reconnu dans son pays, est sollicité par la SIA espagnole pour y créer une section

33 Voir IISG, CNT 88B.

34 Ibidem. 
nationale. Au Royaume-Uni, Emma Goldman35, qui fait partie du conseil d'honneur de la SIA, est sollicitée par Pedro Herrera pour constituer autour d'elle une section britannique ${ }^{36}$. L'existence d'une section de la SIA au Royaume-Uni, est prépondérante, c'est un pays puissant, qui est encore une démocratie, et qui ne possède pas d'organisation libertaire structurée. L'aura d'Emma Goldmann y est importante et la SIA pense qu'elle peut rassembler autour de son nom un certain nombre de militants. En Suède, le mouvement libertaire est beaucoup mieux implanté grâce au syndicat SAC. C'est grâce à John Andersson37, membre fondateur du syndicat et secrétaire de l'AIT, que la section suédoise voit le jour. Lui aussi, comme Emma Goldmann, fait partie du conseil d'honneur de la SIA et se sert de la reconnaissance qu'il a en Suède auprès des militants révolutionnaires pour mettre en place une section nationale de la SIA. En janvier 1938, la section américaine est créée de la même manière, autour du syndicaliste et théoricien allemand exilé aux Etats-Unis, Rudolf Rocker38, lui aussi membre du conseil d'honneur de la SIA39. Si les premières sections actives se créent, grâce à l'aura de militants reconnus, dans des pays où le mouvement est à l'état embryonnaire, ou qui ne possèdent pas de militant libertaire qui fasse l'unanimité autour de lui, la tâche est plus complexe.

Après la première vague de créations, le développement international de la SIA s'étale sur toute l'année 1938. Cette deuxième vague, présente elle aussi des caractéristiques communes dans la façon de créer les nouvelles sections : la plupart du temps, ce sont des militants du MLE en exil, ou des connaissances de ces mêmes militants qui se mettent en contact direct avec le conseil général de la SIA installé à Barcelone, pour solliciter leur aide pour la création d'une section nationale de la SIA. Dans le premier semestre 1938, c'est surtout l'Amérique Latine qui se distingue, avec la création de sections en Argentine, au Chili, au Mexique et en Uruguay40. Dans ces pays hispanophones, où l'implantation des idées libertaires varie d'une région et d'une ville à l'autre, ce sont des militants exilés de la CNT, informés par le biais de leurs anciens camarades, qui créent les sections nationales de la SIA. En Chine, c'est le camarade

\footnotetext{
35 Emma Goldmann (1869-1940), militante anarchiste américaine d'origine russe, est la secrétaire de la section britannique de la SIA et effectue à ce titre un voyage en Catalogne en 1937.

36 Voir IISG, CNT 87C.

37 John Andersson (1887-1967), fondateur et secrétaire de la SAC en 1910, est membre du Conseil International de Solidarité Internationale Antifasciste en 1937. En 1938 il devient secrétaire de l'AIT, poste qu'il occupe jusqu'en 1953. Il est un défenseur acharné de l'anarcho-syndicalisme.

38 Rudolf Rocker (1873-1958), théoricien anarchiste allemand; son ouvrage Nationalisme et culture est l'une des références de la philosophie libertaire. Exilé aux Etats-Unis, il devient membre de la SIA en 1938 à la création de la section nationale de ce pays.

39 Voir IISG, CNT 87C.

40 Ibidem.
} 
Chang-Ching-Chiu, de la ville de Yunnanfu, l'actuelle Kunming, qui a effectué plusieurs séjours en Europe, qui crée une section chinoise de la SIA4․ Aux Pays-Bas, c'est Harm Kolthek ${ }^{42}$, militant anarcho-syndicaliste reconnu, qui fonde la section hollandaise de la SIA43. De même, en Australie, ce sont d'anciens militants de la CNT et de la FAI, exilés après la répression de la grève dans les Asturies en 1934, qui créent des sections à Melbourne et Sidney en mars 193844. En tout, il existe fin juin 1938 treize sections nationales de la SIA.

Durant le deuxième semestre 1938, apparaît une dernière vague de créations de sections internationales, pour lesquelles nous ne possédons que peu de renseignements. Les actes de la réunion internationale de la SIA tenue à Paris le $1^{\text {er }}$ novembre 1938, mentionnent l'existence d'une section polonaise ainsi que de sections palestinienne, canadienne et japonaise en création ${ }^{45}$, mais aucun autre document ne revient dessus. On peut supposer que comme dans les cas de la deuxième vague de création, ces sections avaient déjà un noyau de militants, qui avaient demandé leur rattachement à la SIA et qu'ils n'ont pas continué leurs activités suite à la « retirada » et à la fin de la guerre en Espagne au premier trimestre 1939. A ces sections qui existent sans exister, il faut rajouter deux sections bien réelles, celles de Cuba et Honolulu, qui ne fait pas encore officiellement partie des Etats-Unis ${ }^{46}$.

Au début de l'année 1939, la SIA est une organisation qui revendique des sections dans une vingtaine de pays, réparties sur les cinq continents ${ }^{47}$. En a peine plus d'un an d'existence, le résultat est remarquable, si l'on compare avec la douzaine d'organisations qui forment à ce moment là l'AIT48.

Emanation du MLE, la SIA est destinée à apporter un soutien politique et matériel aux anti-autoritaires espagnols sous couvert d'actions de solidarité. Pourtant, les réalités de la guerre amènent la SIA à évoluer. Que cela soit dans ses objectifs, ses méthodes, cette évolution laisse peu à peu au second plan l'idéologie, pour développer l'acte de solidarité. Mais même en recentrant son action sur la solidarité en faveur des victimes de la guerre, la SIA n'oublie pas que cette aide doit être apportée en priorité à son propre camp. A aucun moment des actions de secours en faveur des nationalistes

\footnotetext{
41 Chang-Ching-Chiu affirme qu'il était en Espagne au moment de la déclaration de la république en 1931 et en France lors des évènements de février 1934. Voir IISG, CNT 87C.

42 Harm Kolthek (1872-1946), écrivain et militant libertaire néerlandais.

43 Voir IISG, ZK 27034.

44 Ibidem.

45 Voir IISG, CNT 88B.

46 Voir IISG, CNT 87C.

47 Voir annexes, document II, pour le détail des sections de la SIA.

48 Voir LORENZO César Marcel, op. cit., p 195.
} 
ne sont envisagées. La SIA réserve ses activités au camp républicain. Cette sélectivité est importante dans la construction de la SIA. Pour des raisons matérielles, idéologiques, le refus de secourir ceux qui ne sont pas de son côté forge son identité. Ce positionnement est impensable aujourd'hui, avec des organisations humanitaires qui prônent la neutralité la plus absolue quand il s'agit de distribuer de l'aide. Même le Secours Populaire Français, héritier du SPFC et du SRI a évolué vers cette neutralité de principe, laissant derrière lui la notion de soutien humanitaire idéologique. La courte existence de la SIA ne nous permet pas de savoir si elle aussi aurait évolué vers cette neutralité. Mais sa construction, ses actions, son activité internationale plaident en faveur d'une organisation qui voulait s’inscrire dans la durée.

Construite par le mouvement libertaire en Espagne et dans les autres pays où elle a une section, la SIA ne se détache à aucun moment de celui-ci. Il lui est difficile de couper les liens qu'elle entretient avec un mouvement qui a tout fait pour la mettre sur les rails et l'imposer dans le paysage de l'aide internationale. Il ne lui est pas non plus possible de refuser l'aide que lui apportent la CNT ou la FAI en Espagne, l'UA en France ou la SAC en Suède. Ces organisations anarchistes qui voient dans la SIA la structure internationale qui leur manque face au développement des organisations communistes. Pourtant, imperceptiblement, la SIA met en place un réseau de solidarité efficace qui ne peut plus laisser planer de doute sur les objectifs qu'elle veut remplir. 


\section{Sources}

Archives de la Préfecture de police de Paris

Cartons : BA/1513, BA/1545, BA/1562, BA/1713, BA/1900

Centre des Archives Contemporaine de Fontainebleau (CAC)

20010216 (ART. 48 -79 : activité des organisations syndicales ouvrières, 1900-1940, ART. 79-92 : suivi du mouvement ouvrier, 1906-1940, ART. 92-119 : activité des partis politiques, 1913-1940)

Institut Français d'Histoire Sociale (IFHS)

Cartons : 14/ASP 91, 14/ASP 1893, 14/ASP 2123

Internationaal Instituut Voor Sociale Geschiedenis (Institut International pour l'Histoire Sociale d'Amsterdam, IISG)

Microfilms : 36B, 41C, 5oB, 64C, 87C, 88B, 91 bis, 99 III, 100B, 100D, 100E, 100F

\section{Bibliographie}

Los médicos y la medicina en la guerra civil española, Madrid, Monografía Beecham, 1986

AUDOIN-ROUZEAU, Stéphane, " Guerre et médecine, quel champs de recherche? », dans Actes de la deuxième journée Guerre et médecine,

URL : < http://www.bium.univ-paris5.fr/histmed/guerre/journee2004/xooaudoin.pdf > [consultée le 20 août 2008]

BERRY, David, « Solidarité Internationale Antifasciste : Les anarchistes français et la guerre civile d'Espagne ", in SAGNES, Jean, CAUCANAS, Sylvie (dir.), Les Français et la guerre d'Espagne, Perpignan, CREPF, 1990, pp 73-88.

BERRY, David, L'anarchisme français et la révolution espagnole 1936-1939, Paris, Alternative Libertaire, 2001

BERRY, David, A History of the French Anarchist Movement, 1917-1945, Westview CT, Greenwood Press, 2002

BRODIEZ, Axelle, Le rôle structurant des guerres dans la genèse de l'humanitaire communiste,

URL: < http://www.bium.univ-paris5.fr/histmed/guerre/journee2007/o7brodiez.pdf > [consultée le 18 juillet 2008)] 
BRODIEZ, Axelle, Le Secours populaire français 1945-20oo Du communisme à l'humanitaire, Paris, Presses de Science Po, 2006

CASTANIER, Tristan, Femmes en exil, mères des camps Elisabeth Eidenbenz et la maternité suisse d'Elne (1939-1944), Canet, Trabucaire, 2008

CIONINI, Valentin, L'Union Anarchiste face à la guerre d'Espagne. De la solidarité politique et matérielle au Front Révolutionnaire, Mémoire de Master 1, Université de Provence, 2007

CIONINI, Valentin, Solidarité Internationale Antifasciste. Une organisation «proto-humanitaire» dans la guerre d'Espagne. 1937-1939, Mémoire de Master, Université de Provence, 2008

COLLOVALD, Annie, LECHIEN, Marie-Hélène (dir.), L'humanitaire, ou le management des dévouements, Rennes, Presses Universitaires de Rennes, 2003

DÍAZ-PLAJA, Fernando, La vida cotidiana en la España de la guerra civil, Madrid, EDAF, 1994

GRISONI, Dominique-Antoine, HERTZOG, Gilles, Les brigades de la mer, Paris, Grasset, 1979

HUGH, Thomas, La guerre d'Espagne, Paris, Robert Laffont, 1997

MARQUÉS, Pierre, Les enfants espagnols réfugiés en France: 1936-1939, P.Marqués, 1993

MARQUÉS, Pierre, La Croix-Rouge pendant la guerre d'Espagne, 1936-1939 - Les missionnaires de l'humanitaire, Paris, L'Harmattan, 2000.

TAITHE, Bertrand, Guerre, médecine et humanité: peut-on écrire une histoire de l'humanitaire?,

URL: < http://www.bium.univ-paris5.fr/histmed/guerre/journee2004/xo4taithe.pdf > [consultée le 2 août 2008] 


\section{* L'auteur}

Valentin Cionini, titulaire d'un Master de l'Université de Provence en 2008, il axe ses recherches sur Solidarité Internationale Antifasciste et le soutien humanitaire du mouvement anarchiste international aux libertaires espagnols. Il est enseignant d'Histoire-Géographie en classes internationales espagnoles.

URL: < http://studistorici.com/progett/autori/\#Cionini >

\section{Per citare questo articolo:}

CIONINI, Valentin, "Solidarité Internationale Antifasciste, ou l'humanitaire au service des idées anarchistes», Diacronie. Studi di Storia Contemporanea: Spagna Anno Zero: la guerra come soluzione, 29/07/2011, URL:< http://www.studistorici.com/2011/07/29/cionini_numero_7/ >

Diacronie Studi di Storia Contemporanea $\beta$ www.diacronie.it

Risorsa digitale indipendente a carattere storiografico. Uscita trimestrale. redazione.diacronie@hotmail.it

Comitato di redazione: Marco Abram - Giampaolo Amodei - Jacopo Bassi - Luca Bufarale - Alessandro Cattunar - Alice De Rensis Barbara Galimberti - Deborah Paci - Fausto Pietrancosta - Martina Sanna - Matteo Tomasoni - Luca Zuccolo 